\title{
Lyophilized Dosage Form Category
}

National Cancer Institute

\section{Source}

National Cancer Institute. Lyophilized Dosage Form Category. NCI Thesaurus. Code C148552.

A type of solid pharmaceutical dose form consisting of a powder or disc formed by freeze-drying of a liquid or semi-solid preparation. When referring to a powder, the use of this term is restricted to veterinary medicines. 